\title{
An Adaptive Interacting Multiple Model Algorithm for Maneuvering Targets Tracking
}

\author{
Shu-Liang WANG, Da-Ping BI and Ming-Yang DU
}

\author{
No. 460, Mount Huangshan Road,Shushan District, Hefei, Anhui, China
}

\begin{abstract}
Keyword: Maneuvering target tracking, IMM, CS model, Model transition probability
Abstract. Current Statistical (CS) model is a good adaptive filtering model for maneuvering target tracking. While, the performance of CS model depends on the maneuvering frequency, and becomes poor when tracking weak maneuvering targets. Firstly, the innovation of the filtering is used to reduce the dependence on the constant of maneuvering frequency. Secondly, in order to improve the performance for weak maneuvering targets, Constant Velocity (CV) model is used to compete with CS model in the framework of Interacting Multiple Model (IMM) algorithm. Thirdly, to avoid the over-competition and enhance the probability of superior model, a time-varying model transition probability function is proposed with the current measure. Simulation results show that this method greatly improves the performance for weak maneuvering targets, and the performance for strong maneuvering targets is similar to that of the CS model.
\end{abstract}

\section{Introduction}

Maneuvering target tracking is widely used for both civilian and military needs, and the first task is to find appropriate motion model. It was pointed that target tracking depends on the effective information extracted from the observation of target[1].A good model is worth thousands of data. Currently, there are two main directions for the modeling of maneuvering targets, one is the single-model algorithm, and the other is the multi-model algorithm. Based on the Singer model[2], the CS model[3] assumed the acceleration in the next moment can only be in the neighborhood of the current acceleration, the current probability of the acceleration obeys modified Rayleigh distribution.

The good performance of CS model for tracking high maneuvering targets has been proved yet. While, the performance of this algorithm depends on the constant of maneuvering frequency. If the constant is set improperly, the tracking performance would become poor. Also, CS model is not proper for weak maneuvering targets, especially for the constant velocity targets [4]-[6]. When the maneuvering is strong, the constant of maneuvering frequency should be set bigger, and vice versa. The maneuvering can be described with the innovation of the filtering. If the innovation becomes larger, the maneuvering becomes stronger. So, we use an innovation function to modify the constant of maneuvering frequency, and a modified CS (MCS) model is proposed.

In [7], Blom and Bar-Shalom proposed an IMM algorithm with Markov transition probability. This algorithm considers the interaction between each model to estimate the target state. The core of the algorithm is to choose the model sets which can include all possible motions. We choose CS model and CV model as the model sets of IMM, which can improve the performance of CS model algorithm for weak maneuvering tracking by the competing of CV model. The Markov transition probability between every model is constant in the traditional IMM algorithm. In fact, the process should be a sojourn-time-dependent process[8]. If the previous state model keeps longer, the probability of this model is higher. So, at a certain moment, if the probability of one model is larger than the previous moment, the transition probability to its own should be strengthened, to another should be reduced. Based on this idea, some researchers[9]-[11] have tried to introduce a weighting factor to adjust the transition probability. In [12], a time-varying transition probability is proposed from Bayesian theory. We use this method to improve the traditional IMM algorithm, in which the transition probability between the models can be adjusted dynamically, and the competition of the improper model is weakened.

The organization of this work is as follows. The adaptive IMM (AIMM) algorithm based on CS model and CV model is presented in Section two. An improved algorithm is given in Section three 
where the MCS model based on the adjustment of maneuvering frequency constant is proposed, the time-varying transition probability is given, and the time-varying adaptive IMM(TAIMM) algorithm based on MCS model and CV model is given. In the Section four, a numerical example compares the performance of the MCS model algorithm with the CS model, the TAIMM algorithm with the MCS model algorithm and the AIMM algorithm. In the last Section, the conclusion is given.

\section{Target Tracking Model}

\subsection{Motion Model}

\section{a). Target State Equation}

The target's discrete state equation can be expressed as

$$
\mathbf{X}^{(i)}(k)=\boldsymbol{\Phi}^{(i)}(k \mid k-1) \mathbf{X}^{(i)}(k-1)+\mathbf{U}(k-1) \bar{a}^{(i)}(k-1)+\mathbf{W}^{(i)}(k-1)
$$

Where $i=1$ means non-maneuvering motion model; $i=2$ means maneuvering motion model. $\boldsymbol{\Phi}$ is the state transition matrix and $\mathbf{U}$ is the input control matrix. $\bar{a}$ is the mean value of current acceleration during the sample period $(k-1) T$. W is Gaussian white noise.

When the target is non- maneuvering, CV model is used, the target state vector can be expressed as $\mathbf{X}^{(1)}(k)=\left[r^{k}, i_{-}, r^{k}\right.$ and $i \quad$ are the range and velocity of the target. $\boldsymbol{\Phi}^{(1)}(k \mid k-1)=\left[\begin{array}{cc}1 & \Delta t \\ 0 & 1\end{array}\right]$ is the state transition matrix, $\Delta t$ is the interval of the observation, the mean of acceleration $\bar{a}^{(1)}=0$, dynamic noise $\mathbf{W}^{(1)}(k-1) \sim N\left(0, \mathbf{Q}^{(1)}\right), \mathbf{Q}^{(1)}$ is the covariance of system noise.

When the target is maneuvering, CS model is used, then the target state vector can be expressed as $\mathbf{X}^{(2)}(k)=\left[r^{k}, i^{\cdots} \cdots, r^{k}, i\right.$ and ${ }^{*} \quad$ are the range, velocity and acceleration of the target. The mean of acceleration $\bar{a}^{(2)} \neq 0$, dynamic noise $\mathbf{W}^{(2)}(k-1) \sim N\left(0, \mathbf{Q}^{(2)}\right)$, CS model can adaptively track maneuvering target from the expression $\mathbf{Q}^{(2)}=2 \alpha \sigma_{a}^{2} \mathbf{Q}_{0}$, where $\alpha$ is the constant of maneuvering frequency, the expression of $\boldsymbol{\Phi}^{(2)}(k \mid k-1), \mathbf{U}(k-1), \sigma_{a}^{2}$ and $\mathbf{Q}_{0}$ can be found in[3]. From the equation $\mathbf{Q}^{(2)}=2 \alpha \sigma_{a}^{2} \mathbf{Q}_{0}$, we can know that the performance of CS model algorithm depends on the constant of maneuvering frequency, if the constant is set improperly, the tracking performance would become poor.

\section{b).Target Measurement Equation}

If the measurement data is only for the position, the measurement equation is

$$
\mathbf{Y}(k)=\mathbf{H}^{(i)}(k) \mathbf{X}^{(i)}(k)+\mathbf{V}(k)
$$

Where $\mathbf{H}^{(1)}(k)=\left[\begin{array}{ll}1 & 0 \\ 0 & 1\end{array}\right], \mathbf{H}^{(2)}(k)=\left[\begin{array}{lll}1 & 0 & 0 \\ 0 & 1 & 0\end{array}\right], \mathbf{V}(k)$ is Gaussian white noise with zero mean value and its covariance matrix is $\mathbf{R}(k)$.

\subsection{Adaptive Interacting Multiple Models (AIMM)}

The steps of IMM algorithm are input interacting, model conditional filtering, model probability updating and estimate fusion. The IMM algorithm block diagram based on the interaction between $\mathrm{CS}$ and $\mathrm{CV}$ models is as follows:

\section{a). Input Interacting}


Assuming that transition probability is $P_{i j}, \hat{X}^{j}(k-1 \mid k-1)$ is the state estimation from filter $j$ at time $k-1, \quad P^{j}(k-1 \mid k-1)$ is corresponded to error covariance, $m_{j}(k-1)$ is the probability of model $j$ at time $k-1$, and $i, j=1,2$, so two filters inputs are calculated as

$$
\begin{aligned}
& \hat{X}^{o j}(k-1 \mid k-1)=\stackrel{\circ}{i=1}^{2} \hat{X}^{i}(k-1 \mid k-1) m_{|| j}(k-1 \mid k-1) \\
& \hat{P}^{o j}(k-1 \mid k-1)=\stackrel{a}{i=1}_{2}^{2}\left\{P^{i}(k-1 \mid k-1)+\left[\hat{X}^{i}(k-1 \mid k-1)-\hat{X}^{o j}(k-1 \mid k-1)-\hat{X}^{o j}(k-1 \mid k-1)\right]\right. \\
&
\end{aligned}
$$

Where

$$
\begin{aligned}
& m_{i \mid j}(k-1 \mid k-1)=\frac{1}{\dot{C}_{j}} P_{i j} m_{i}(k-1) \\
& \dot{C}_{j}=\stackrel{\AA}{i=1}_{\stackrel{2}{2} P_{i j} m_{i}(k-1)}
\end{aligned}
$$

\section{b). Model Conditional Filtering}

The input of model $j$ at time $k$ are $\hat{X}^{o j}(k-1 \mid k-1)$ and $\hat{P}^{o j}(k-1 \mid k-1)$, and after Kalman filter, the output are $\hat{X}^{j}(k \mid k-1)$ and $\hat{P}^{j}(k \mid k-1)$.

\section{c). Model Probability Updating}

If the filtering innovation of model $j$ is $v_{j}(k)$, and $\mathbf{S}_{j}(k)$ is covariance, assuming that the innovation obeys the Gaussian distribution. So the likelihood function is as follows

$$
\Lambda_{j}(k)=\frac{1}{\sqrt{\left|2 \pi S_{j}(k)\right|}} \exp \left\{-\frac{1}{2}\left[v_{j}^{T}(k) S_{j}^{-1}(k) v_{j}(k)\right]\right\}
$$

Where

$$
\begin{array}{r}
v_{j}(k)=Y(k)-H_{j}(k) \hat{X}^{j}(k \mid k-1) \\
S_{j}(k)=H_{j}(k) P^{j}(k \mid k-1) H_{j}^{T}(k)+R_{j}(k)
\end{array}
$$

The model probability is updated as

$$
\begin{gathered}
\mu_{j}(k)=\frac{1}{C} \Lambda_{j}(k) \bar{C}_{j} \\
C=\sum_{i=1}^{2} \Lambda_{i}(k) \bar{C}_{i}
\end{gathered}
$$

\section{d). Estimate Fusion}

$$
\begin{gathered}
\hat{X}(k \mid k)=\sum_{i=1}^{2} \hat{X}^{i}(k \mid k) \mu_{i}(k) \\
P(k \mid k)=\sum_{i=1}^{2}\left\{\left[\hat{X}^{i}(k \mid k)-\hat{X}(k \mid k)\right]\left[\hat{X}^{i}(k \mid k)-\hat{X}(k \mid k)\right]^{T}\right\} \mu_{i}(k)+\sum_{i=1}^{2} P^{i}(k \mid k) \mu_{i}(k)
\end{gathered}
$$




\section{Time-varying Adaptive IMM Algorithm (TAIMM)}

\subsection{Modified CS model algorithm (MCS)}

From equation 4, the covariance matrix of CS model algorithm is related to maneuvering frequency constant. In order to obtain better performance for tracking, a larger constant should be chosen for high maneuvering and a smaller one for weak maneuvering (if the constant is equal to zero, CS model is turned into CV model). The innovation of the target in CS model filtering reflects the maneuvering index of the target. The target innovation at time $k$ is

$$
v(k)=Y(k) \quad H(k) X(k+h
$$

The dynamic adjustment function is

$$
f(v(k))=1-\exp \left(-\frac{(v(k))^{\kappa}}{\mu S(k)}\right)
$$

Where $S(k)$ is the corresponding covariance of innovation and $\mu=2$. When $v(k) \leq 3 \sqrt{R}, \kappa=2$. When $v(k)>3 \sqrt{R}, \kappa=4 . R$ is measurement noise covariance.

When the target is maneuvering with a larger acceleration, $f(v(k)) \rightarrow 1$. When the target is maneuvering with a smaller acceleration, $f(v(k)) \rightarrow 0$. At each sampling moment, $\alpha$ changes into $\alpha^{\prime}=\alpha f(v(k))$, and all the values associated with $\alpha$ have changed accordingly.

\subsection{Time-Vary Transition Probability of the Model}

In traditional IMM algorithm, the model switching is assumed to be governed by a Markov chain with the pre-determined transition probabilities matrix such as

$$
\left.\left.p_{i j}(k-1) \square \quad, \quad\right) \mid M_{i}(k-1)\right\}=p_{i j}
$$

Where $M_{j}(k), j=1,2, \ldots \mathrm{N}$, and it means that transition probability is constant. It has nothing to do with the sampling time $k$. The probabilities should be considered as a function of sample time, and corrected by the latest measurement. So transition probabilities are redefined as follows

$$
\left.\left.p_{i j}(k-1) \square \quad, \quad\right) \mid M_{i}(k-1), Y^{k-1}\right\}
$$

Where $Y^{k-1}=\left\{Y_{1}, Y_{2}, \ldots \quad \ldots \quad\right.$ is the measurement before $k-1$ sample period, and its one-step estimation transition probability is as follow

$$
\left.\left.p_{i j}(k \mid k-1) \square \quad, \quad\right) \mid M_{i}(k-1), Y^{k}\right\}
$$

It can also be regarded as time $k-1$ of transition probability posteriori estimation, based on Bayesian theory, the transition probability of prediction can be expressed as

$$
p_{i j}(k \mid k-1)=P\left\{M_{j}(k) \mid M_{i}(k-1), Y^{k}\right\}=\frac{1}{c_{i}(k-1)} p_{i j}(k-1) \lambda_{i j}(k)
$$

$p_{i j}(k-1)$ is the transition probability at time $k-1, \lambda_{i j}(k)$ is the likelihood of measurement conditioned on the models over two recent sample period and measurement sequence $Y^{k-1}$ and $Y_{k}$.

$$
\lambda_{i j}(k)=f\left[Y_{k} \mid M_{j}(k), M_{i}(k-1), Y^{k-1}\right]
$$


$f\left(\square\right.$ is the probability density function, and $c_{i}(k-1)=\sum_{j=1}^{N} p_{i j}(k-1) \lambda_{i j}(k)$ is the normalized factor. Regardless of the measurement before time $k-1$, the measurement value $Y_{k-1}$ can be approximately estimated with filtering value of model $i$ at time $k-1$

$$
\begin{gathered}
Y^{k-1} \approx \hat{X}^{i}(k-1 \mid k-1) \\
\lambda_{i j}(k) \approx f\left[Y_{k} \mid M_{j}(k), M_{i}(k-1), \hat{X}^{i}(k-1 \mid k-1)\right]
\end{gathered}
$$

If the function obey Gaussian distribution, then

$$
\lambda_{i j}(k) \approx N\left[Y_{k} ; H_{k}^{j} \hat{X}^{i j}(k \mid k-1), H_{k}^{j} P^{i j}(k \mid k-1)\left(H_{k}^{j}\right)^{T}+R_{k}^{j}\right]
$$

$\hat{X}^{i j}(k \mid k-1)$ and $\hat{P}^{i j}(k \mid k-1)$ are state estimation and covariance matrix which use the filtering value and covariance of the model $j$ to estimate the model $i$.Suppose that in a sampling time period the transition probability is constant, so

$$
p_{i j}(k) ? p_{i j}(k \mid k \quad 1)
$$

\section{Simulation Results}

In order to test the effectiveness of the new algorithm, Monte Carlo trials are used. The root mean square error (RMSE) of the state estimation is used to evaluate the performance

$$
\operatorname{RMSE}(k)=\sqrt{\frac{1}{\mathrm{~N}} \sum_{i=1}^{\mathrm{N}}\left[x^{i}(k \mid k)-\hat{x}(k \mid k)\right]^{2}}
$$

Where $\mathrm{N}$ is the number of Monte Carlo trials, and $x^{i}(k \mid k)$ is the real target state at sampling time $k$ and $\hat{x}(k \mid k)$ is the estimation of target state.

\subsection{Simulation Condition}

Target state transition probabilities matrix is a Markov chain with two models, which are the CS model $(\alpha=1 / 20)$ and CV model. The covariance of measurement is $\mathrm{R}=5000 \mathrm{~m}^{2}, a_{-\max }=-80 \mathrm{~m} / \mathrm{s}^{2}$, $a_{\max }=80 \mathrm{~m} / \mathrm{s}^{2}, \mathrm{~N}=100$, the initial value of transition probabilities matrix is $\left[\begin{array}{ll}0.9 & 0.1 \\ 0.1 & 0.9\end{array}\right]$. Simulation of target trajectory is divided into three stages. The initial value of target state is $(0,200 \mathrm{~m} / \mathrm{s}, 0)$. The sampling period is 1 second.

Stage I: From $t=0 \mathrm{~s}$ to $t=30 \mathrm{~s}$, target moves at a constant velocity $\left(a=0 \mathrm{~m} / \mathrm{s}^{2}\right)$.

Stage II: From $t=31 \mathrm{~s}$ to $t=60 \mathrm{~s}$, target moves at a constant acceleration $\left(a=40 \mathrm{~m} / \mathrm{s}^{2}\right)$.

Stage III: From $t=61 \mathrm{~s}$ to $t=100 \mathrm{~s}$, target moves at a constant acceleration $\left(a=-20 \mathrm{~m} / \mathrm{s}^{2}\right)$.

\section{a). CS model algorithm and the MCS model algorithm}

Fig. 1(a) and Fig. 1(b) give the velocity and acceleration RMSE of CS model and MCS model. 


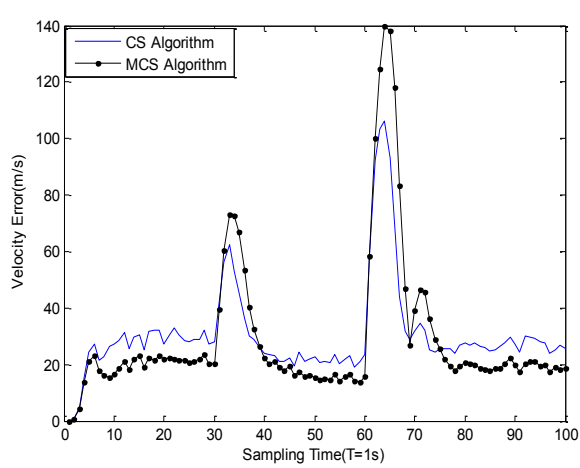

(a) The velocity RMSE of two algorithms

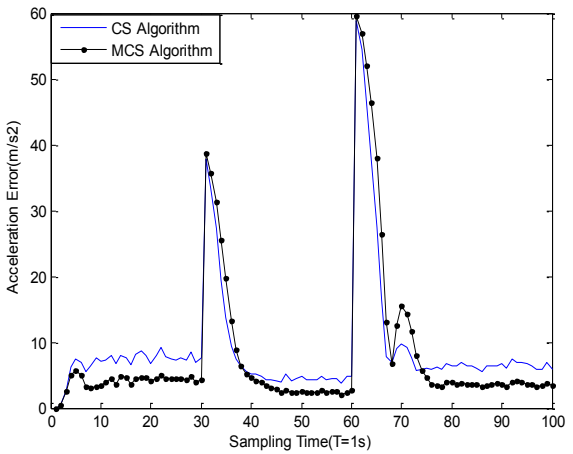

(b) The acceleration RMSE of two algorithms

Fig. 1 Comparisons of the mean square root error of two algorithms

\subsection{Simulation Analysis}

As is shown in Fig.1, when CS model algorithm is used, the performance for strong maneuvering target (Stage II and Stage III) is better than for the weak maneuvering target (Stage I). The constant of maneuvering frequency is set to $1 / 20$, so the system variance is corresponding to this constant. In MCS model algorithm, the maneuvering frequency is changed dynamically with the maneuvering trend. Thus, the system variance is adjusted dynamically. Although this algorithm converges slower than CS model algorithm at the time of acceleration varying sharply, it greatly improves the tracking performance in the steady tracking stage.

This modified CS model algorithm obtains better performance for tracking both strong maneuvering and weak maneuvering target. While, the performance for tracking weak maneuvering target is still worse than that of tracking the strong maneuvering target.

\section{a.). MCS model algorithm, AIMM algorithm and TAIMM algorithm}

Fig. 2 gives the velocity and acceleration RMSE of MCS model algorithm, AIMM algorithm, and TAIMM algorithm.

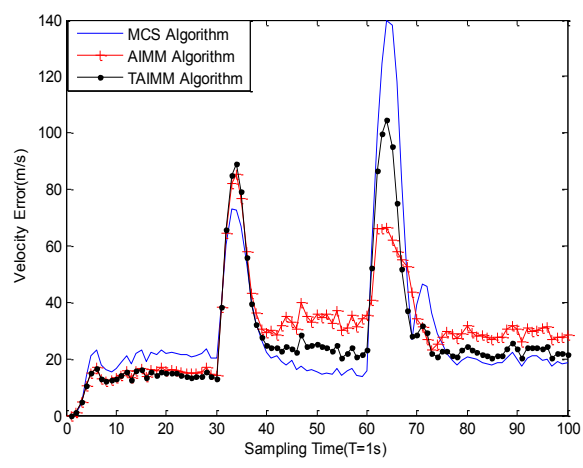

(a) The velocity RMSE of three algorithms

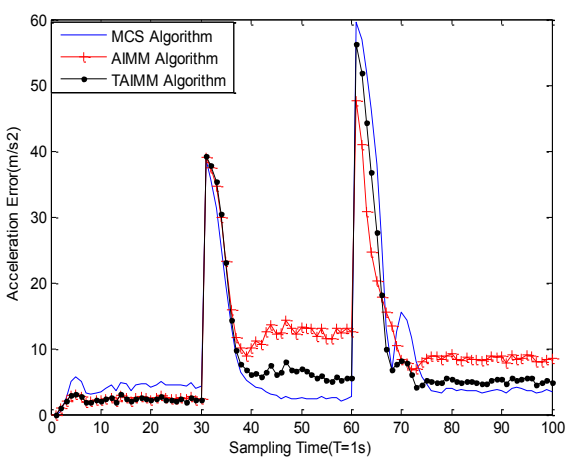

(b) The acceleration RMSE of three algorithms

Fig. 2 Comparisons of the mean square root error of three algorithms

Fig. 3 (a) and (b) are probabilities distributions of the model selection for AIMM algorithm and TAIMM algorithm in the three stages respectively. 


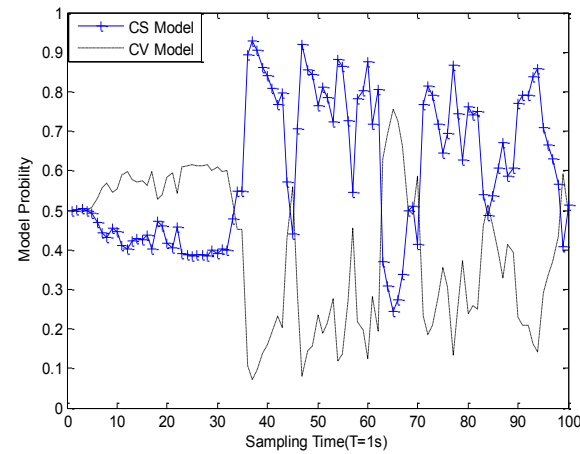

(a) Model probabilities with AIMM

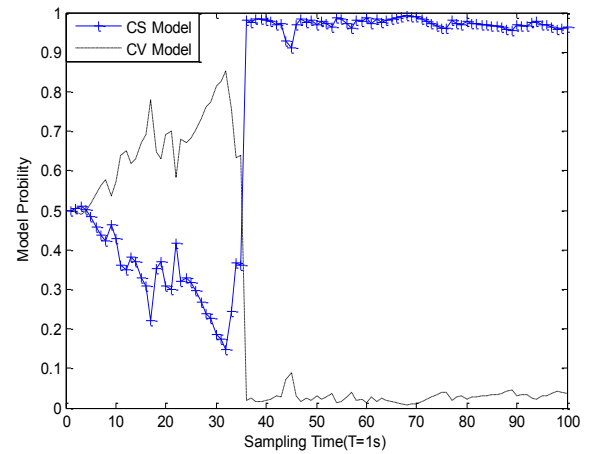

(b) Model probabilities with TAIMM

Fig. 3 Probabilities of the model selection for two algorithms

\subsection{Simulation Analysis}

As is shown in Fig. 2, the MCS model still has a larger tracking error for weak maneuvering targets (Stage I).With AIMM algorithm, the performance for tracking weak maneuvering target is improved due to the competition of the $\mathrm{CV}$ model, and the convergence at the time of acceleration varying sharply is also improved. While, the performance for tracking strong maneuvering target becomes worse. With TAIMM algorithm, the model transition probability is adaptive with the current measure. The model with larger model probability is more likely to transfer to itself, and reduce influence about undesirable competition. As is shown in Fig. 3, with TAIMM algorithm, the probability is distinguished with each other well for the adaptive transition probability. TAIMM algorithm is a tradeoff between MCS model algorithm and the AIMM algorithm. It can obtain good performance for tracking weak maneuvering target as CV model, and also obtain similar performance for tracking strong maneuvering target as MCS model.

\section{Conclusion}

A time-varying adaptive IMM algorithm is proposed in this paper. The model sets only include two sets, MCS model and CV model, which would have certain value for tracking maneuvering target in engineering.

\section{References}

[1]LI X R, Jilkov V P. Survey of Maneuvering Target Tracking-Part I: Dynamic Models[J]. IEEE Transactions on aerospace and electronic systems, 2003, 39(4):1333-1364

[2]Singer R A. Estimation Optimal Tracking Filter Performance for Manned Maneuvering Targets[J], IEEE Transactions on Aerospace and Electronic Systems, 1970, 6(4), 473-483.

[3]Zhou H, Kumar K. A Current Statistical Model and Adaptive Algorithm For Estimating Maneuvering Targets[J], AIAA Journal, Guidance, Control Dynamics , 1984, 7(5):596-602.

[4]Yongjian Yang, Xiaoguagn Fan. A new parameters adaptively adjusting method of current statistical model[J], Proceeding of the 2015 IEEE International Conference on Information and Automation, 2015:1738-1742.

[5] Wei Sun, Yongjian Yang. Adaptive Maneuvering Frequency Method of Current Statistical Model[J], IEEE/CAA Journal of Automatic Sinica , 2016:1-7.

[6] Wen Li, Qingdong Li. Maneuvering Acceleration Assisted Attitude Algorithm Design Based on Fuzzy Adaptive Kalman Filter[C], Proceedings of 2014 IEEE Chinese Guidance, Navigation and Control Conference,2014:1501-1505.

[7]Blom H A P, Bar-Shalom Y. The Interacting Multiple Model Algorithm for Systems with 
Markovian Switching Coefficients[J], IEEE Transactions on Automatic Control, 1988, 33(8):780-783.

[8]L. Campo, P. Mookerjee, Bar-Shalom Y. State estimation for Systems with a Sojoum-Time-Dependent Markov Swithing Model[J]. IEEE Trans on Auto. Control,1991,36(2):238-243.

[9]Eun Y, Jeon D. Fuzzy interence-based dynamic determination of IMM mode transition probability for multi-radar tracking[C]. International Conference on Information Fusion. IEEE, 2013:1520-1525.

[10] Xin Bi, Wei Wang, JieGao and Jinsong Du. The improved IMM tracking algorithm for high-speed maneuvering target[C]. Intelligent detection and laboratory equipment. 2015:1-3.

[11]GUO Z, DONG C Y, CAI Y L, et al. Time-varying transition probability based IMM-SRCKF algorithm for maneuvering target tracking[J]. Systems Engineering and Electronics, 2015,37(1):24-30.

[12] ZHENG D K, WANG S Y.A new interacting multiple model algorithm for maneuvering target tracking based on adaptive transition probability updating[C]. IET International Radar Conference. 2015:5-5. 